the study by Katona \& Roth (1985), cited by Dr Kumar, also reports, in the authors' own words, 'an increased frequency of HPA axis abnormality' in schizoaffective depression.

We agree that our study would have been more complete if we had measured the adrenal gland size. Indeed, measuring hormonal levels would have been an important addition to the study. We will take this advice into consideration in our future studies.

Finally, we support Dr Kumar's view that hyperactivity of the HPA axis occurs in a large number of conditions associated with stress. Indeed, we suggested in our paper that glucocorticoid resistance could be the molecular mechanism by which stress induces HPA axis hyperactivity in patients with different mental disorders (see Discussion, p. 8). We never suggested that this biological abnormality could have any diagnostic value. However, we believe that measuring specific biological markers can give us further insight into the pathological mechanisms affecting the brains (and the bodies) of our patients.

Banki, C. M., Bissette, G., Arato, M., et al (1987) CSF corticotropin-releasing factor-like immunoreactivity in depression and schizophrenia. American Journal of Psychiatry, 144, 873-877.

Brooks, B. S., el Gammal, T., Allison, J. D., et al (1989) Frequency and variation of the posterior pituitary bright signal on MR images. American Journal of Neuroradiology, 10, 943-948.

Herz, M. I., Fava, G. A., Molnar, G., et al (1985) The dexamethasone suppression test in newly hospitalised schizophrenic patients. American Journal of Psychiatry 142, 127-129.

Ismail, K., Murray, R. M., Wheeler, M. J., et al (1998) The dexamethasone suppression test in schizophrenia. Psychological Medicine, 28, 3II-3I7.

Katona, C. L. \& Roth, M. (1985) The dexamethasone suppression test in schizo-affective depression. Journal of Affective Disorders, 8, 107-112.

Lammers, C. H., Garcia-Borreguero, D., Schmider, J., et al (1995) Combined dexamethasone/ corticotropin-releasing hormone test in patients with schizophrenia and in normal controls: Il. Biological Psychiatry, 38, 803-807.

Pariante, C. M., Vassilopoulou, K., Velakoulis, D., et a (2004) Pituitary volume in psychosis. British Journal of Psychiatry, 185, 5-10.

Sachar, E. J., Kanter, S. S., Buie, D., et al (1970) Psychoendocrinology of ego disintegration. American Journal of Psychiatry, 126, 1067-1078.

C. M. Pariante Division of Psychological Medicine, Box PO5I, Institute of Psychiatry, King's College London, I Windsor Walk, Denmark Hill, London SE5 8AF, UK. E-mail: spjucmp@iop.kcl.ac.uk

\section{CBT for psychosis}

Although the study by Tarrier et al (2004) appears to be methodologically more rigorous than the similar study of cognitivebehavioural therapy (CBT) in post-acute schizophrenia by Turkington et al (2002), I am a little confused by the authors' conclusions. After clearly demonstrating no superior effect for CBT over supportive counselling on measures of symptom reduction and relapse rates, the authors conclude their paper by stating that they 'suggest that the optimum psychosocial management of early schizophrenia would include a combination of CBT and family intervention'. Would it be rude to suggest that the authors take into account their own findings before making such a statement? It is also more than a little irritating that the authors refer to their sample as being diagnosed with 'early schizophrenia' throughout the paper. Examining the inclusion criteria for this study shows that patients were included who had schizophreniform disorders, delusional disorders and unspecified psychoses. Regarding the criterion for 'early', most clinicians would define the duration of schizophrenia from symptom onset to commencement of treatment (hence the concept of the duration of untreated psychosis). Unless the authors specify illness duration, the criterion of 'early' cannot be asserted. Thus, this appears to be a study of patients within 2 years of their first episode of nonaffective psychoses and not those with early schizophrenia per se.

Tarrier, N., Lewis, S., Haddock, G., et al (2004) Cognitive-behavioural therapy in first-episode and early schizophrenia: 18-month follow-up of a randomised controlled trial. British Journal of Psychiatry, 184, 231-239.

Turkington, D., Kingdon, D. \& Turner, T. (2002) Effectiveness of a brief cognitive-behavioural intervention in the treatment of schizophrenia. British Journal of Psychiatry, 180, 523-527.

A. J. Mitchell Brandon Mental Health Unit, Gwendolen Road, Leicester LE5 4PW, UK. E-mail: alex.mitchell@leicspart.nhs.uk

Authors' reply: Dr Mitchell professes both confusion and irritation at our report of the follow-up results of the SoCRATES study. I will attempt to clarify. When implemented with standard care, CBT has consistently been found to result in clinical benefits in terms of symptom relief compared with standard care alone. This has been found in post-acute illness, as with the reported study, and chronic phases (Pilling et al, 2002). Cognitive-behavioural therapy is a structured psychological treatment usually implemented from a manual, which makes it relatively amenable to 'roll-out'; CBT has less impact on relapse rates. Family interventions have been shown to have the benefit of significantly reducing relapse rates (Pilling et al, 2002). Thus, combining both CBT and family interventions would appear to be the most parsimonious way of capitalising on these developments to improve patient care by reducing symptoms of psychosis and reducing risk of subsequent relapse. In addition, both service users and carers have been increasingly vocal in wishing a greater range of interventions to be made available, including both psychological treatments and assistance for families. It is regrettable that Dr Mitchell's comments implicitly appear to wish to deny them these further options. With respect to his comment on our inclusion criteria for the trial, a first episode of psychosis resulting in treatment by mental health services is an event that can be identified with reasonable accuracy (at least, much more accurately than emergence of symptoms prior to this). As $80 \%$ of study participants had first-episode illness using this criterion, the use of the term 'recent onset' is not unreasonable. The inclusion of those suffering from schizophreniform psychosis, delusional disorders and unspecified psychosis reflects clinical practice and conforms to convention on large pragmatic clinical trials in having broad inclusion criteria to aid generalisation (see Johnson, 1998).

Johnson, T. (1998) Clinical trials in psychiatry: background and statistical perspective. Statistical Methods in Medical Research, 7, 209-234.

Pilling, S., Bebbington, P., Kuipers, E., et al (2002) Psychological treatments in schizophrenia: I. Metaanalysis of family interventions and cognitive behaviour therapy. Psychological Medicine, 32, 763-782.

N. Tarrier, G. Haddock, S. Lewis School of Psychiatry and Behavioural Sciences, Education and Research Centre (2nd floor), Wythenshawe Hospital, Southmoor Road, Manchester M23 9LT, UK. E-mail: nicholas.tarrier@man.ac.uk

\section{Depression intervention in resource-poor regions}

Depression is recognised as a common problem in developing countries and it is one of the most important causes of morbidity and mortality (Patel et al, 2001). Lack of 